Notas y comunicaciones 


\section{Trabajos colaborativos en torno a la Alfabetización Estadística}

Collaborative works

on Statistical Literacy

Liliana Mabel Tauber

\section{Introducción}

Aunque hace algún tiempo que no presentamos información en la sección Notas y Comunicaciones, consideramos que es esta una buena oportunidad para retomar el diálogo a través de la misma.

En esta ocasión, y en consonancia con la temática planteada en el Editorial y en el presente número de Yupana, queremos aportarles información relacionada con distintas experiencias que vienen llevándose adelante desde hace al menos una década en relación al trabajo colaborativo de profesores, investigadores y estudiantes en torno a la Educación Estadística. Es así que, brevemente, presentaremos información asociada con tres instancias, dos de las cuales ya tienen un desarrollo importante y otra que, aunque más reciente, permite abrir un espacio de discusión que esperamos mantener a través del tiempo. ¡Los invitamos a conocer estas experiencias y también a participar en ellas!

Nombre del trabajo: Trabajos colaborativos en torno a la Alfabetización Estadística Nombre y apellido del autor: Liliana Mabel Tauber

Dirección completa: Azcuénaga 4265, Santa Fe, Argentina

Lugar de trabajo: Universidad Nacional del Litoral, Facultad de Humanidades y Ciencias Ciudad Universitaria, Paraje El Pozo, Santa Fe, Argentina

Teléfono de contacto: Celular: +54-343 - 4479790

Dirección electrónica: estadisticamatematicafhuc@gmail.com 


\section{El Proyecto Internacional de Alfabetización Estadística (ISLP)}

Como su nombre lo indica, este Proyecto se sostiene y desarrolla gracias al esfuerzo desinteresado de muchos profesores y estudiantes que, alrededor de todo el mundo, están interesados, preocupados y ocupados por difundir una Educación Estadística que permita desarrollar el pensamiento crítico y la cultura ciudadana.

La misión del Proyecto Internacional de Alfabetización Estadística (ISLP por sus siglas en inglés) es apoyar, crear y participar en actividades y promoción de la Alfabetización Estadística en todo el mundo. Para facilitar la comunicación entre los diversos proyectos y países que participan, se desarrolló una página web: https://iase-web.org/islp/, la cual cumple la función de ser un foro donde aquellos interesados en la Alfabetización Estadística, puedan reunirse (en un sentido virtual), intercambiar ideas y necesidades, información y recursos y aprender a difundir la alfabetización estadística en sus comunidades.

El ISLP está apoyado por la Asociación Internacional de Educación Estadística y por el Instituto Internacional de Estadística (IASE e ISI respectivamente, por sus siglas en inglés) y se compone de varios proyectos, cada uno de ellos enfocado en un área de la Alfabetización Estadística. Cada proyecto es coordinado por voluntarios expertos (el coordinador del proyecto) que compilan recursos, recolectan información que se publica en la página web y ejecutan actividades dedicadas a aumentar la visibilidad de la Alfabetización Estadística en su área de especialización. El Director de ISLP supervisa todos los proyectos y propicia distintas actividades que busca nuclear a todos ellos y a la comunidad mundial.

Dentro de estos proyectos se destacan la edición de un boletín (o newsletter) semetral en el que se brinda información de distintas acciones sobre Alfabetización Estadística, llevadas adelante en distintos países del mundo. Particularmente, en el último boletín de mayo de 2020 se brinda información sobre actividades llevadas adelante en 2019 en Argentina y, en ediciones anteriores, también pueden encontrarse artículos de otros países lati- noamericanos. Para acceder a las distintas ediciones del boletín, pueden ingresar a: https://iase-web. org/islp/Publications.php.

Al interior de ISLP, una de las actividades que se lleva a cabo desde 2007, en esa ocasión como experiencia piloto, es la Competencia o Competición Internacional de Alfabetización Estadística, la cual se centra en la elaboración y presentación de un tema de investigación elegido por grupos de estudiantes a través de un póster que se presenta de manera virtual. Se busca que el póster muestre aportes originales realizados por los estudiantes con la orientación de un profesor responsable. La información estadística que los estudiantes deben procesar puede estar basada en datos que recolectan los mismos estudiantes o simplemente pueden utilizarse bases de datos publicadas y realizar distintos análisis estadísticos que busquen dar respuestas a alguna pregunta de investigación que se hayan planteado los mismos estudiantes. Para más información sobre las características de esta Competencia pueden consultar en: https:// iase-web.org/islp/Competitions.php.

Los objetivos principales de la Competencia Internacional de Alfabetización Estadística son:

- Promover acciones educativas que favorezcan el desarrollo de la Alfabetización Estadística.

- Desarrollar habilidades de indagación y divulgación científica a través de un póster en el que se utilicen herramientas estadísticas.

- Fomentar el intercambio de experiencias entre los diferentes actores que intervienen en un proceso de indagación científica.

- Favorecer la consolidación de comunidades de aprendizaje, que contribuyan a estrechar lazos colaborativos entre docentes y alumnos.

La Competencia se realiza en el marco de los Congresos bianuales de ISI, por lo que cada instancia permite exponer los trabajos seleccionados de cada país participante en distintas partes del mundo, en las que se realizan dichos congresos. Esta com- 
pentencia consta de dos etapas que se desarrollan durante los dos años que dura; la primera etapa es la instancia nacional, en la que los coordinadores de cada país se encargan de realizar las inscripciones y asesoramiento de los interesados en participar y, luego, junto a un jurado evaluador, seleccionan los trabajos que más se adecuan a los objetivos y temática de cada convocatoria. Esos trabajos seleccionados en cada país, pasan a participar de la competencia internacional y, en el Congreso bianual del ISI, se seleccionan los trabajos ganadores a nivel mundial. Todos los estudiantes de Educación Secundaria y Superior que estén interesados pueden participar de manera grupal en la instancia nacional y, podrán participar de la instancia internacional si son seleccionados en su país. El único requisito es que cada grupo de estudiantes tengan un profesor guía que sea el responsable de orientarlos y de brindarles apoyo en la búsqueda de información y en el análisis de los datos. La inscripción es totalmente gratuita, tanto en la instancia nacional como en la internacional. Para acceder a la información de los coordinadores de cada país, pueden ingresar a: https://iase-web. org/islp/People.php?p=Country_Coordinators

Y si desean más información para la Competencia 2020-2021, pueden consultar: https://iase-web. org/islp/Poster_Competition_2020-2021.php. Particularmente, en Argentina, la información de contacto de los coordinadores es la siguiente:

- Liliana Tauber. Facultad de Humanidades y Ciencias. Universidad Nacional del Litoral. E-mail: estadisticamatematicafhuc@gmail.com

- Juan José Sosa. Universidad Nacional del Nordeste. E-mail:sosajuanj@gmail.com

\section{La Red Latinoamericana de Investigación en Educación Estadística (RELIEE)}

Esta Red es otra instancia de trabajo colaborativo que año a año va creciendo, la cual permite el contacto y el intercambio entre profesores de Matemática y de Estadística que se desempeñan en distintos niveles educativos e investigadores especializados en la Educación Estadística.

La Red Latinoamericana de Investigación en Educación Estadística (RELIEE), se gestó en la Reunión Latinoamericana de Matemática Educativa (RELME), realizada en Buenos Aires, en el año 2013, y desde ese momento ha tomado como punto de reunión de sus integrantes, las reuniones anuales de RELME (https://clame-relme.org/), participando principalmente en un Grupo de Discusión permanente así como en actividades que varían cada año.

Los principales propósitos de la RELIEE se resumen en los siguientes ítems:

- Generar redes de intercambio y colaboración en grupos de trabajo;

- Promover el desarrollo de la autonomía de sus miembros;

- Promover espacios de diálogo, debate y reflexión; - Incluir y promover la participación de la comunidad docente y social.

Además de las reuniones anuales en los grupos de discusión antes mencionados se ha desarrollado una página web: https://reliee.weebly.com/ a partir de la cual se divulgan distintas acciones que lleva a cabo RELIEE o algunos de los grupos que la componen. Asimismo, se brindan datos sobre revistas o páginas que ofrecen información asociada a la Educación Estadística y, en la actualidad, se están delineando nuevas instancias de intercambio entre sus miembros de manera virtual. La inscripción a la Red es totalmente gratuita y puede realizarse a través de un formulario Google que está disponible en la página de la Red, en el siguiente enlace: https://reliee.weebly.com/inscripcion-a-la-red. html. Actualmente, la Red está compuesta por más de 110 miembros provenientes de todos los países latinoamericanos y de España.

Consideramos que puede ser una buena oportunidad para conocer a nuevos miembros que puedan proponer distintos proyectos o acciones que permitan mejorar la Educación Estadística en cada una de nuestras regiones, por lo que los invitamos a formar parte de esta comunidad. 


\section{Jornadas Argentinas de Educación Estadística (JAEE)}

El 1 y 2 de noviembre de 2019, se realizaron en la Facultad de Humanidades y Ciencias (FHUC) de la Universidad Nacional del Litoral (UNL), en la ciudad de Santa Fe (Argentina), las I Jornadas Argentinas de Educación Estadística (JAEE).

Dichas Jornadas han sido un espacio de intercambio de propuestas didácticas y divulgación e investigación de la Educación Estadística entre investigadores, profesores y estudiantes de distintos niveles educativos. En las mismas se propició el intercambio de experiencias entre estudiantes que han desarrollado proyectos estadísticos con profesores de educación primaria, secundaria y superior. Asimismo, se desarrollaron conferencias y un foro de discusión con invitados internacionales en modalidad virtual, del cual participaron: Dra. Carmen Batanero (España), Dr. Armando Albert (México), Dr. Hugo Alvarado (Chile) y Dra. Claudia Vásquez (Chile), junto a especialistas argentinos que desarrollaron 4 cursos y 5 talleres destinados a la formación continua de profesores.

Las temáticas de las conferencias y foros fueron: - Conectando la probabilidad a la vida cotidiana.

- La Estadística no es solo análisis de los datos.

- Observación de clases de probabilidad en el aula de Educación Primaria: Una herramienta para fortalecer la formación inicial docente.

- La Red Latinoamericana de Investigación en Educación Estadística: Una comunidad de práctica que fomenta la Educación Estadística en Latinoamérica.

El evento más importante en esta instancia fue el Workshop: «Los estudiantes nos cuentan sus experiencias estocásticas», en la que los principales ponentes fueron los estudiantes, quienes previamente presentaron un video de 5 minutos elaborados por ellos mismos, en el que realizaron un spot publicitario con el cual presentaban el trabajo estadístico que habían realizado en sus escuelas. A continuación, respondieron a preguntas del auditorio conformado por otros estudiantes y por los docentes asistentes, y también nos contaron sus pareceres y sentimientos generados luego de haber transitado la experiencia.

Fue el momento de mayor riqueza de todas las Jornadas, ya que fue una oportunidad para conocer los distintos momentos que debieron atravesar los estudiantes (junto al profesor a cargo) para poder obtener los datos, pensar cómo analizarlos y sacar conclusiones y también pudimos conocer sus preocupaciones y sus deseos en torno a la distintas problemáticas que abordaron.

Otro punto relevante de las experiencias presentadas por los estudiantes fue el hecho de que en la mayoría de los trabajos, a raíz del estudio realizado, los estudiantes tuvieron la posibilidad de acercar sus conclusiones a distintos organismos gubernamentales $\mathrm{y}$, actualmente, están desarrollando un trabajo conjunto para mejorar distintos aspectos de su propio entorno social o medioambiental.

Las temáticas de los trabajos presentados fueron:

- Pedaleando $\mathrm{x}=$ derechos

- Estadística en la electromecánica

- Contaminación del ferrocarril

- Uso de las tecnologías

- El respeto: ¿valor principal en la vida cotidiana?

- Educación vial

- Siguiendo la Huella

Se prevé realizar de manera anual estas jornadas y mantener dos espacios: uno para la formación y actualización de los profesores y el más importante, que es el espacio destinado a los estudiantes porque consideramos que son los principales promotores de la Educación Estadística y ellos son los que inspiran a los profesores. En la página: https://estocasticos. weebly.com/, encontrarán información asociada a las Jornadas y también al Grupo de investigación en Educación Estadística de la UNL, cuyas integrantes han sido las promotoras de las mismas. 